\title{
Critical Velocity Determined by a Non-Exhaustive Method in Subjects With COPD
}

\author{
Marceli Rocha Leite MSc, Juliana Souza Uzeloto MSc, Bruna Spolador de Alencar Silva MSc, \\ Ana Paula Coelho Figueira Freire MSc, Fabiano Francisco de Lima MSc, \\ Eduardo Zapaterra Campos PhD, Diego Giulliano Destro Christofaro PhD, \\ Carlos Augusto Kalva-Filho MSc, Dionei Ramos PhD, and Ercy Mara Cipulo Ramos PhD
}

\begin{abstract}
BACKGROUND: Exercise training is an effective and low-cost tool for COPD rehabilitation. Thus, validating a non-exhaustive method to determine a patient's anaerobic threshold would be a very interesting tool in practical settings. We aimed to test the reproducibility of critical velocity values determined in a non-exhaustive manner $\left(\mathrm{CV}_{\mathrm{NE}}\right)$ and tolerance for exercise performed at this physiological marker in subjects with COPD. METHODS: Twelve subjects with COPD were evaluated in this cross-sectional study. The study was divided into 2 phases: Phase A consisted of reproducibility of $\mathrm{CV}_{\mathrm{NE}}$ for subjects with COPD, and Phase $\mathrm{B}$ determined the tolerance for exercise tests performed at this physiological marker. RESULTS: The proposed protocol for determining $\mathbf{C V}_{\mathrm{NE}}$ presented fair reproducibility according to blood lactate concentrations (intraclass correlation coefficient $=\mathbf{0 . 5 8}$ ). However, we cannot consider that the tests were reproducible due to the wide CI (0.03-0.85). Furthermore, moderate and strong correlations were observed between the $\mathbf{C V}_{\mathrm{NE}}$ determined by lactate concentration, by oxygen consumption $\left(\dot{\mathrm{V}}_{\mathbf{O}_{2}}\right)(\mathrm{r}=\mathbf{0 . 6 6})$, and by heart rate $(r=0.88)$. However, a high limit of agreement was observed $\pm 1.45 \mathrm{~km} / \mathrm{h}$ and $\pm 1.55 \mathrm{~km} / \mathrm{h}$, respectively. In the tolerance for exercise test, none of the subjects presented lactate destabilization, whereas 3 tests were interrupted by oxygen saturation decline and 1 test was interrupted by hemodynamic instability. CONCLUSIONS: Although most of the subjects presented good tolerance for exercise at $\mathbf{C V}_{\mathrm{NE}}$, fair levels of reproducibility were observed in subjects with $\mathrm{COPD}$, even using lactate. Thus, the use of $\mathbf{C V}_{\mathrm{NE}}$ determined through double 3-min efforts should be considered with caution. Key words: COPD; exercise; critical velocity. [Respir Care 2018;63(3):319-325. ( 2018 Daedalus Enterprises]
\end{abstract}

\section{Introduction}

COPD is known not only for its pulmonary effects but also for systemic manifestations (eg, muscle weakness, inflammation). ${ }^{1}$ Several studies have demonstrated that exercise training is an effective and low-cost tool during COPD rehabilitation, improving muscular strength, exercise tolerance, and quality of life. ${ }^{2}$

\footnotetext{
Drs D Ramos and EMC Ramos, Messrs Leite and de Lima, and Ms Uzeloto, de Alencar Silva, and Freire are affiliated with the Department of Physiotherapy, Universidade Estadual Paulista, Presidente Prudente, São Paulo, Brazil. Dr Campos is affiliated with the Department of Physical Education, Universidade Estadual Paulista, Rio Claro, São Paulo, Brazil. Dr Christofaro is affiliated with the Department of Physical Education, Universidade Estadual Paulista, Presidente Prudente, São Paulo, Brazil. Mr Kalva-Filho is affiliated with the Department of Rehabilitation and Functional Performance, Universidade de São Paulo, Ribeirão Preto, São Paulo, Brazil.
}

\footnotetext{
This work was supported by Conselho Nacional de Desenvolvimento Científico e Tecnológico, grant no. 301632/2015-4. The authors have reported no other conflicts of interest.
}

Correspondence: Marceli Rocha Leite MSc, Roberto Simonsen, 305, Jardim das Rosas - Presidente Prudente, São Paulo, Brazil. E-mail: marcelirocha@hotmail.com

DOI: $10.4187 /$ respcare.05637 
The prescription of exercise intensity for this population should be individualized instead of adopting general parameters, such as intensity calculations according to heart rate ${ }^{3,4}$ or fatigue scale. ${ }^{5}$ In this context, the prescription of exercise through maximum tests such as the cardiopulmonary exercise test ${ }^{6,7}$ is common in clinical practice..$^{4,8}$ Two aerobic parameters can be determined using these methods: the maximal intensity achieved, which is frequently concomitant with maximal oxygen consumption, and the anaerobic threshold. ${ }^{9}$ Although several studies prescribe exercise training using the maximal intensity achieved, the anaerobic threshold is an important physiological index that demarcates the intensity at which systemic acidosis increases. ${ }^{10}$ However, determination of this physiological marker is limited in patients with COPD mainly by the exhaustive characteristics of the cardiopulmonary exercise, in which dyspnea and hemodynamic alterations can be observed. ${ }^{11}$ Gas exchange should be also monitored during the effort, increasing the cost of evaluation and discomfort of patients who are required to wear a mask. ${ }^{6}$

For these reasons, a non-exhaustive method to determinate the anaerobic threshold would be a very interesting tool in practical settings. In this context, Chassain $^{12}$ determined the anaerobic threshold using several double non-exhaustive efforts. The protocol establishes that the participant needs to perform 2 submaximal efforts, at the same intensity, with $90 \mathrm{~s}$ of passive recovery between them. Blood lactate concentrations (Lac) are determined after each effort, and the difference between them is calculated ( $\Delta \mathrm{Lac})$. This procedure is repeated 3-4 times using different intensities, and the anaerobic threshold is calculated as the intensity that presents a balance between the efforts (ie, $\Delta \mathrm{Lac}=0$ ), which is determined using simple linear regression. The use of the mathematical procedure has led some authors to call the anaerobic threshold determined by Chassain as critical velocity determined in a non-exhaustive manner $\left(\mathrm{CV}_{\mathrm{NE}}\right) \cdot{ }^{13-15}$

Therefore, the use of Chassain's procedure is a practical tool to determine $\mathrm{CV}_{\mathrm{NE}}$, mainly through the use of non-exhaustive efforts. Considering that $\mathrm{CV}_{\mathrm{NE}}$ is theoretically the intensity of physiological balance, it is possible that other parameters can be used for its determination without blood samples, such as heart rate or oxygen consumption $\left(\dot{\mathrm{V}}_{\mathrm{O}_{2}}\right)$. However, no studies have tested the reproducibility or tolerance for exercise performed at $\mathrm{CV}_{\mathrm{NE}}$, which does not allow its use in monitoring and prescribing exercise training for patients with COPD. Thus, we aimed to test the reproducibility of $\mathrm{CV}_{\mathrm{NE}}$ and the tolerance for exercise performed at this physiological marker in subjects with COPD.

\section{QUICK LOOK}

\section{Current knowledge}

Appropriate exercise prescription for patients with COPD enhances the results of the intervention. Prescription of exercise intensity should be individualized instead of adopting general parameters, such as calculations through heart rate or fatigue scale. Exercise prescription using maximum tests is common in clinical practice. However, this method is limited in patients with COPD, mainly because of its exhaustive characteristics, in which dyspnea and hemodynamic alterations can be observed.

\section{What this paper contributes to our knowledge}

The proposed protocol to determine critical velocity determined in a non-exhaustive manner presented fair reproducibility in subjects with COPD, and most subjects presented good tolerance for exercise at maximum intensity. This protocol may be a new tool for exercise prescription, saving the patient from performing at maximum physical exertion.

\section{Methods}

\section{Subjects}

For this study, 12 subjects with COPD from a physical therapy clinic of São Paulo State University in Brazil were evaluated. To participate in the study, the inclusion criteria were to have the results of spirometry tests and medical diagnosis of the disease as recommended by the Global Initiative for Obstructive Lung Disease (GOLD). ${ }^{16}$ Exclusion criteria were current smoking, use of home oxygen therapy, presence of comorbidities or musculoskeletal disorders that prevented execution of the experimental protocol, and unstable COPD (ie, exacerbations and medication changes in the previous $30 \mathrm{~d}$ ). All procedures were approved by the ethics committee of the institution (CAAE: 01114912.0.0000.5402), and all subjects signed a consent form.

\section{Study Design}

This cross-sectional study was performed over 10 days. The study was divided into 2 phases (Fig. 1). In Phase $\mathrm{A}$, the reproducibility of $\mathrm{CV}_{\mathrm{NE}}$ was tested; during Phase $\mathrm{B}$, the tolerance for exercise performed at $\mathrm{CV}_{\mathrm{NE}}$ was assessed. The first day involved subject identification, anthropometric measurements, spirometry, and the 6-min walk test (6MWT). On the other days, the subjects were 


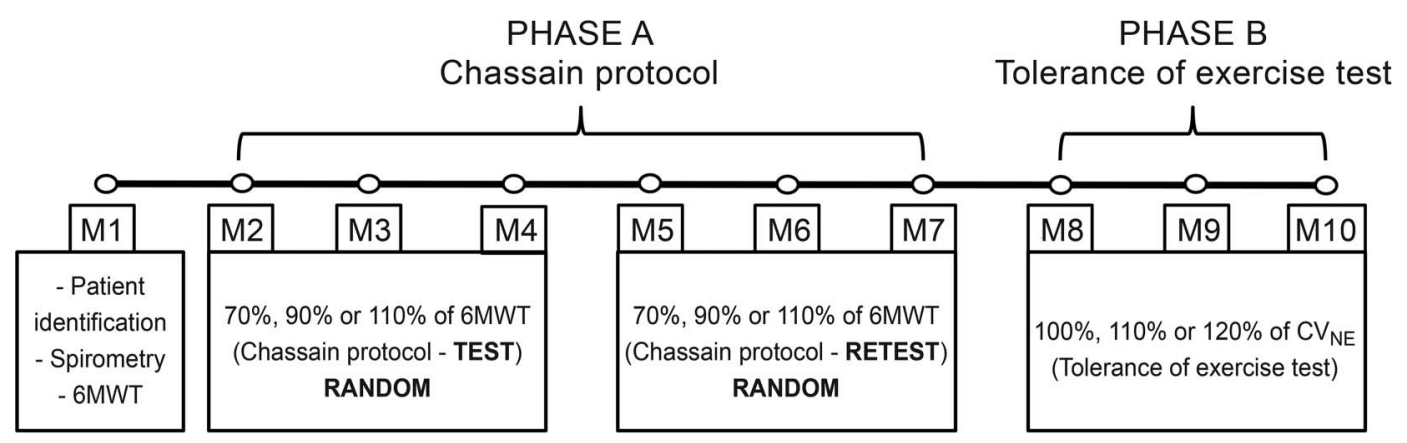

Fig. 1. Experimental protocol. $\mathrm{M}=$ moment, $6 \mathrm{MWT}=6-\mathrm{min}$ walk test, $\mathrm{CV}_{\mathrm{NE}}=$ critical velocity determined in a non-exhaustive manner.

randomly assigned to 3 exercise sessions with an intensity of $70 \%, 90 \%$, and $110 \%$ of the mean speed achieved in the 6MWT. ${ }^{13}$ In each session, double efforts were performed at the same intensity, with a duration of $180 \mathrm{~s}$ each, separated by an interval of $90 \mathrm{~s}$. Subsequently, the subjects repeated the 3 speeds, also in random order, to assess the reproducibility of the $\mathrm{CV}_{\mathrm{NE}}$ values. The tests were performed at intervals of at least $24 \mathrm{~h}$.

Finally, $3 \mathrm{~d}$ of assessment were performed for exercise tolerance at $\mathrm{CV}_{\mathrm{NE}}$. Phase $\mathrm{B}$ was carried out to simulate a training prescription held at the $\mathrm{CV}_{\mathrm{NE}}$ intensity. Subjects performed a session of exercise at speeds of $100 \%, 110 \%$, and $120 \%$ of the $\mathrm{CV}_{\mathrm{NE}}$ obtained in the first test performed during phase $\mathrm{A}$. The sessions were conducted on 3 different days, at least $24 \mathrm{~h}$ apart. The subjects maintained normal administration of bronchodilators during evaluation days. Because the subjects normally used this medication, it is understood that their usual daily condition was evaluated.

Subjects were instructed to eat a light meal $2 \mathrm{~h}$ before testing, to avoid strenuous exercise the day before the evaluations, to avoid caffeine for $12 \mathrm{~h}$ before the test, and to wear appropriate and comfortable clothing for the exercise.

\section{Anthropometric Evaluation, Pulmonary Function Assessment, and 6MWT}

Body mass was measured on a digital scale (R/I 200, Welmy, Brazil). Height was measured using a stadiometer (Sanny, Brazil). Anthropometric measurements were obtained according to Lohman et al. ${ }^{17}$ Body mass index was also calculated.

Pulmonary function was assessed according to spirometry tests, using an MIR-Spirobank 3.6 version spirometer (MIR, Italy). Interpretations of the results were performed according to the American Thoracic Society and European Respiratory Society guidelines. ${ }^{18}$ Normality values were related to the Brazilian population. ${ }^{19}$

The functional exercise capacity was assessed with the 6MWT according to the guidelines of the American Tho- racic Society. ${ }^{20}$ The experimental values obtained were analyzed according to values provided by Enright and Sherrill. ${ }^{21}$

\section{Phase A: Chassain Protocol}

Participants performed 3 sessions of exercise at $70 \%$, $90 \%$, and $110 \%$ of the average speed achieved in 6MWT. In each session, double efforts were performed at the same intensity, with duration of $180 \mathrm{~s}$ each, separated by a passive interval of $90 \mathrm{~s}$. All tests were performed on a treadmill (ATL Inbramed, Inbrasport, Porto Alegre, Brazil). Heart rate was monitored continuously (Polar Electro, S810i, Finland), and oxygen consumption, carbon dioxide production, and ventilation were measured (VO2000, MGC Diagnostics, Saint Paul, Minnesota).

Blood samples $(25 \mu \mathrm{L})$ were collected from the earlobe, at rest and after each effort, to determine lactate concentration and examined with a sport lactate analyzer (YSI 1500, YSI, Yellow Springs, Ohio). Peripheral oxygen saturation $\left(\mathrm{S}_{\mathrm{pO}_{2}}\right)$ was verified with pulse oximetry (PM100C, NewTech, China) and subjective perception of dyspnea and fatigue was assessed using the Borg scale. ${ }^{22}$

The mean heart rate, oxygen consumption $\left(\dot{\mathrm{V}}_{\mathrm{O}_{2}}\right)$ observed in the final $30 \mathrm{~s}$, and Lac were determined at the end of each effort. The difference between the second and first efforts determined the $\Delta$ lactate, $\Delta$ heart rate, and $\Delta$ $\dot{\mathrm{V}}_{\mathrm{O}_{2}}$.

A linear adjustment was performed between (lactate, heart rate, and $\dot{\mathrm{V}}_{\mathrm{O}_{2}} ; x$ axis) and the exercise intensity $(70 \%$, $90 \%$, and $110 \%$ of 6MWT; $y$ axis). Thus, the CVNE was assumed as the $y$-intercept of this adjustment. ${ }^{12}$

Example: Effort at $70 \%$ of 6MWT: $\Delta$ lactate $=-0.48$ $\mathrm{mM}$; speed $=4.1 \mathrm{~km} / \mathrm{h}$.

Effort at $90 \%$ of 6MWT: $\Delta$ lactate $=0.26 \mathrm{mM}$; speed $=5.2 \mathrm{~km} / \mathrm{h}$.

Effort at $110 \%$ of $6 \mathrm{MWT}: \Delta$ lactate $=0.93 \mathrm{mM}$; speed $=6.4 \mathrm{~km} / \mathrm{h}$.

Thus, $x$ axis was composed of $\Delta$ lactate values, and the $y$ axis was composed of speed values. Adjusting this re- 


\section{Non-Exhaustive Method to Determine Anaerobic Threshold}

lationship using a linear regression procedure, the following equation was obtained:

Speed $=(1.6287 \times \Delta$ lactate $)+4.8479$

Considering that the linear coefficient is the value corresponding to the lactate stabilization (ie, $\Delta$ lactate $=0$ ), the $\mathrm{CV}_{\mathrm{NE}}$ was assumed for this subject to be $4.8479 \mathrm{~km} / \mathrm{h}$.

\section{Phase B: Tolerance for Exercise Test}

The exercise tolerance test was carried out to simulate a training prescription held at the $\mathrm{CV}_{\mathrm{NE}}$ intensity. These sessions were conducted to observe the kinetics of lactate concentration, that is, to observe whether subjects would present lactate destabilization during the progression of the sessions.

The participants performed 3 constant efforts on a treadmill, each lasting $30 \mathrm{~min}$, simulating a training session. The intensities of the effort were $100 \%, 110 \%$, and $120 \%$ of the $\mathrm{CV}_{\mathrm{NE}}$ conducted on 3 different days, at least 24 hours apart.

The lactate, heart rate, $\mathrm{S}_{\mathrm{pO}_{2}}$, Borg fatigue, and Borg dyspnea were determined at rest, after $10 \mathrm{~min}$, and after $30 \mathrm{~min}$.

\section{Statistical Treatment}

The normality of the data were confirmed with the Shapiro-Wilk test. The comparison of the means of $\mathrm{CV}_{\mathrm{NE}}$ between the test and retest was analyzed with the dependent $t$ test. The intraclass correlation coefficient, the coefficient of variation, and typical error ${ }^{23}$ were verified using lactate, $\dot{\mathrm{V}}_{\mathrm{O}_{2}}$, and heart rate in the test and retest. The intraclass correlation coefficients were defined as $<0.40=$ poor; $0.40-0.60=$ fair; $0.60-$ $0.74=$ good; and $>0.75=$ strong. $^{24}$ The effect size classes were defined according to calculated d values: small $(\mathrm{d} \leq 0.2)$, moderate $(\mathrm{d}=0.5)$, or large $(\mathrm{d} \geq 0.8)] .25$ Comparisons between $\mathrm{CV}_{\mathrm{NE}}$ in the test determined through the lactate concentration, $\dot{\mathrm{V}}_{\mathrm{O}_{2}}$, and heart rate were tested with 1-way analysis of variation.

Correlations were performed between the $\mathrm{CV}_{\mathrm{NE}}$ determined with lactate, with $\dot{\mathrm{V}}_{\mathrm{O}_{2}}$, and with heart rate with the Spearman test. Agreement between $\mathrm{CV}_{\mathrm{NE}}$ in the test determined through the lactate, $\dot{\mathrm{V}}_{\mathrm{O}_{2}}$, and the heart rate was tested with the Bland-Altman plot. ${ }^{26}$ The statistical program used was SPSS version 15.0.

\section{Results}

\section{Phase A: Chassain Protocol}

Twelve subjects with COPD were included in this study. The anthropometric, functional, and initial spirometric data from the subjects are presented in Table 1.
Table 1. Anthropometric, Functional, and Spirometric Data of the Study Group

\begin{tabular}{lc}
\hline \hline Male subjects, $n(\%)$ & $7(58.33)$ \\
Age, y & $66.5 \pm 8.91$ \\
BMI, kg/cm ${ }^{2}$ & $25.4 \pm 3.5$ \\
FVC & $2.54 \pm 0.83$ \\
FEV 1 & $1.51 \pm 0.59$ \\
FEV $_{1} / \mathrm{FVC}$ & $59.33 \pm 11.17$ \\
GOLD I, $n$ & 1 \\
GOLD II, $n$ & 8 \\
GOLD III, $n$ & 3 \\
GOLD IV, $n$ & 0 \\
6MWD, m & $547.3 \pm 75.23$ \\
6MWT speed, km/h & $5.45 \pm 0.78$ \\
\hline$N=12$ subjects. Data expressed as mean \pm SD unless otherwise noted. & \\
BMI $=$ body mass index & \\
GOLD $=$ Global Initiative for Obstructive Lung Disease & \\
6MWD $=6$-min walk distance & \\
6MWT $=$ 6-min walk test & \\
\hline
\end{tabular}

Table 2 shows the values obtained by linear regression to determine $\mathrm{CV}_{\mathrm{NE}}$. No differences were observed between test and retest in lactate $(P=.89)$ or heart rate $(P=.74)$, whereas significant differences were observed in $\dot{\mathrm{V}}_{\mathrm{O}_{2}}(P=.02)$. Fair intraclass correlation coefficient values were observed for determination of $\mathrm{CV}_{\mathrm{NE}}$ using lactate (intraclass correlation coefficient $=0.58$ ). However, we cannot consider that the tests were reproducible due to the wide CI (0.03-0.85). Additionally, poor agreement was observed using heart rate. Moderate typical error values were detected in $\mathrm{CV}_{\mathrm{NE}}$ for all variables. No differences were observed when comparing $\mathrm{CV}_{\mathrm{NE}}$ determined by lactate, $\dot{\mathrm{V}}_{\mathrm{O}_{2}}$, and heart rate $(P=.34)$.

During the efforts at $110 \%$ (in Chassain's procedures), 2 subjects required oxygen supplementation during the test, and 2 subjects required oxygen supplementation during the retest. Furthermore, significant correlations were observed between $\mathrm{CV}_{\mathrm{NE}}$ determined by lactate, $\mathrm{CV}_{\mathrm{NE}}$ determined by $\dot{\mathrm{V}}_{\mathrm{O}_{2}}(\mathrm{r}=0.66, P=.01)$, mean error $=-0.54$, limits of agreement $\pm 1.45 \mathrm{~km} / \mathrm{h}$ (Fig. $2 \mathrm{~A}$ ); and heart rate $(\mathrm{r}=0.88, P<.001)$, mean error $=0.09$, limits of agreement $\pm 1.55 \mathrm{~km} / \mathrm{h}$ (Fig. 2B).

\section{Phase B: Tolerance for Exercise Test}

In the tolerance for exercise test, none of the subjects displayed lactate destabilization (difference of $1 \mathrm{mM}$ in lactate concentration), as there was no difference in the variables of heart rate $(P=.54), \mathrm{S}_{\mathrm{pO}_{2}}(P=.23)$, Borg dyspnea $(P=.65)$, and Borg fatigue $(P=.94)$ at different intensities $\left(100 \%, 110 \%\right.$, and $120 \%$ of $\left.\mathrm{CV}_{\mathrm{NE}}\right)$. However, 3 tests were interrupted by a decline of oxygen saturation, and 1 test was interrupted by hemodynamic instability at $120 \%$ (Fig. 3). 
Non-Exhaustive Method to Determine Anaerobic Threshold

Table 2. Variables Derived from Linear Adjustment, Required for Determining $\mathrm{CV}_{\mathrm{NE}}$ Using Lactate Concentration, $\dot{\mathrm{V}}_{\mathrm{O}_{2}}$, and Heart Rate

\begin{tabular}{|c|c|c|c|c|c|c|c|}
\hline & Test & Retest & Effect Size & Typical Error & $\begin{array}{l}\text { Coefficient of } \\
\text { Variation, } \%\end{array}$ & $\begin{array}{c}\text { Intraclass Correlation } \\
\text { Coefficient }\end{array}$ & $95 \% \mathrm{CI}$ \\
\hline \multicolumn{8}{|l|}{$\mathrm{CV}_{\mathrm{NE}}, \mathrm{km} / \mathrm{h}$} \\
\hline Lactate & $4.43 \pm 0.94$ & $4.47 \pm 0.88$ & -0.02 & 0.60 & 13.42 & 0.58 & $0.03-0.85$ \\
\hline$\dot{\mathrm{V}}_{\mathrm{O}_{2}}$ & $4.98 \pm 0.83$ & $3.94 \pm 1.61 *$ & 0.37 & 0.85 & 18.44 & 0.40 & $-0.19-0.78$ \\
\hline Heart rate & $4.35 \pm 1.49$ & $4.48 \pm 0.77$ & -0.06 & 1.01 & 22.76 & 0.29 & $-0.32-0.72$ \\
\hline \multicolumn{8}{|c|}{$\begin{array}{l}=12 \text { subjects. Values expressed as mean } \pm \mathrm{SD} \text { and percentage unless otherwise noted. } \\
{ }^{*} \text { Dependent } t \text { test, } P=.027 . \\
\mathrm{CV}_{\mathrm{NE}}=\text { non-exhaustive critical velocity } \\
\text { Lactate = lactate concentration } \\
\dot{\mathrm{V}}_{\mathrm{O}_{2}}=\text { oxygen consumption }\end{array}$} \\
\hline
\end{tabular}
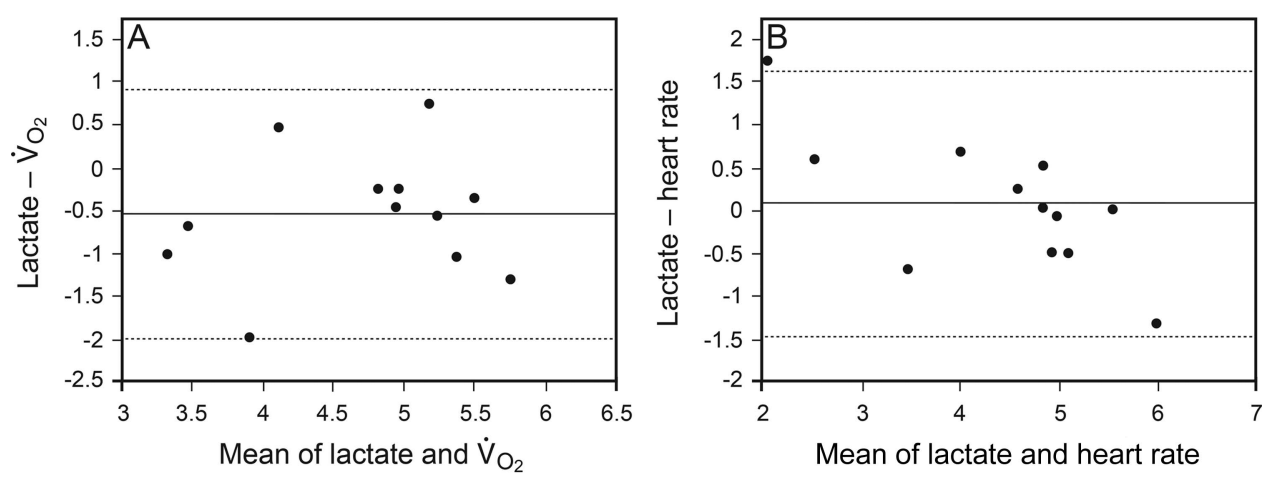

Fig. 2. Bland-Altman plot of the difference between the critical velocity determined in a non-exhaustive manner ( $\left.C V_{N E}\right)$ determined by lactate concentration. $C V_{\mathrm{NE}}$ determined by oxygen consumption $\left(\dot{V}_{\mathrm{O}_{2}}\right)(\mathrm{A})$, and $\mathrm{CV}_{\mathrm{NE}}$ determined by heart rate $(\mathrm{B})$. Center lines denote means, and dotted lines show \pm 1.96 SD.

\section{Discussion}

The main findings of this study demonstrated that the protocol proposed by Chassain ${ }^{12}$ for determining $\mathrm{CV}_{\mathrm{NE}}$ presented fair reproducibility through lactate in subjects with COPD. Despite moderate correlations between the $\mathrm{CV}_{\mathrm{NE}}$ determined by lactate or the $\mathrm{CV}_{\mathrm{NE}}$ determined by $\dot{\mathrm{V}}_{\mathrm{O}_{2}}$ and heart rate, substituting the lactate for these variables is not recommended due to the high limit of agreement observed. In the exercise tolerance testing, no subjects presented lactate destabilization; however, 3 tests were interrupted by a decline of oxygen saturation, and 1 test was interrupted by hemodynamic instability.

\section{Phase A: Chassain Protocol}

Despite the fact that comparison of $\mathrm{CV}_{\mathrm{NE}}$ values between test and retest according to lactate and heart rate demonstrated fair reproducibility with small values of effect size in subjects with COPD, we cannot consider that the tests were reproducible due to the large CI. Also, differences were observed between test and retest in the values of critical velocity determined by $\dot{\mathrm{V}}_{\mathrm{O}_{2}}$.
While lactate is considered a standard method to determine $\mathrm{CV}_{\mathrm{NE}}$, it is an invasive method. Thus, reproducibility, correlation, and agreement analysis between lactate, heart rate, and $\dot{\mathrm{V}}_{\mathrm{O}_{2}}$ are important because they are noninvasive variables. One possible explanation for why $\dot{\mathrm{V}}_{\mathrm{O}_{2}}$ was not reproducible may be due to the relationship between ventilation and the relative intensity to determine parameters related to aerobic capacity, which is a method often used to establish the metabolic thresholds in healthy individuals. ${ }^{27}$ However, the observed changes in the ventilation values during incremental exercise may result in ventilatory inefficiency in individuals with COPD. ${ }^{28}$ This fact could compromise the relationship between ventilation and intensity to determine metabolic thresholds.

Considering that Lac is a standard method to determine critical velocity, the correlation and agreement analysis of lactate with heart rate and $\dot{V}_{\mathrm{O}_{2}}$ were not satisfactory. Despite the moderate correlation with $\dot{\mathrm{V}}_{\mathrm{O}_{2}}$ $(r=0.66)$ and a high correlation with heart rate $(r=0.88)$, these results should be interpreted with caution. The high agreement limit of heart rate $( \pm 1.55 \mathrm{~km} / \mathrm{h})$ and $\dot{\mathrm{V}}_{\mathrm{O}_{2}}$ $( \pm 1.45 \mathrm{~km} / \mathrm{h})$ make it unfeasible to substitute lactate for these variables. 

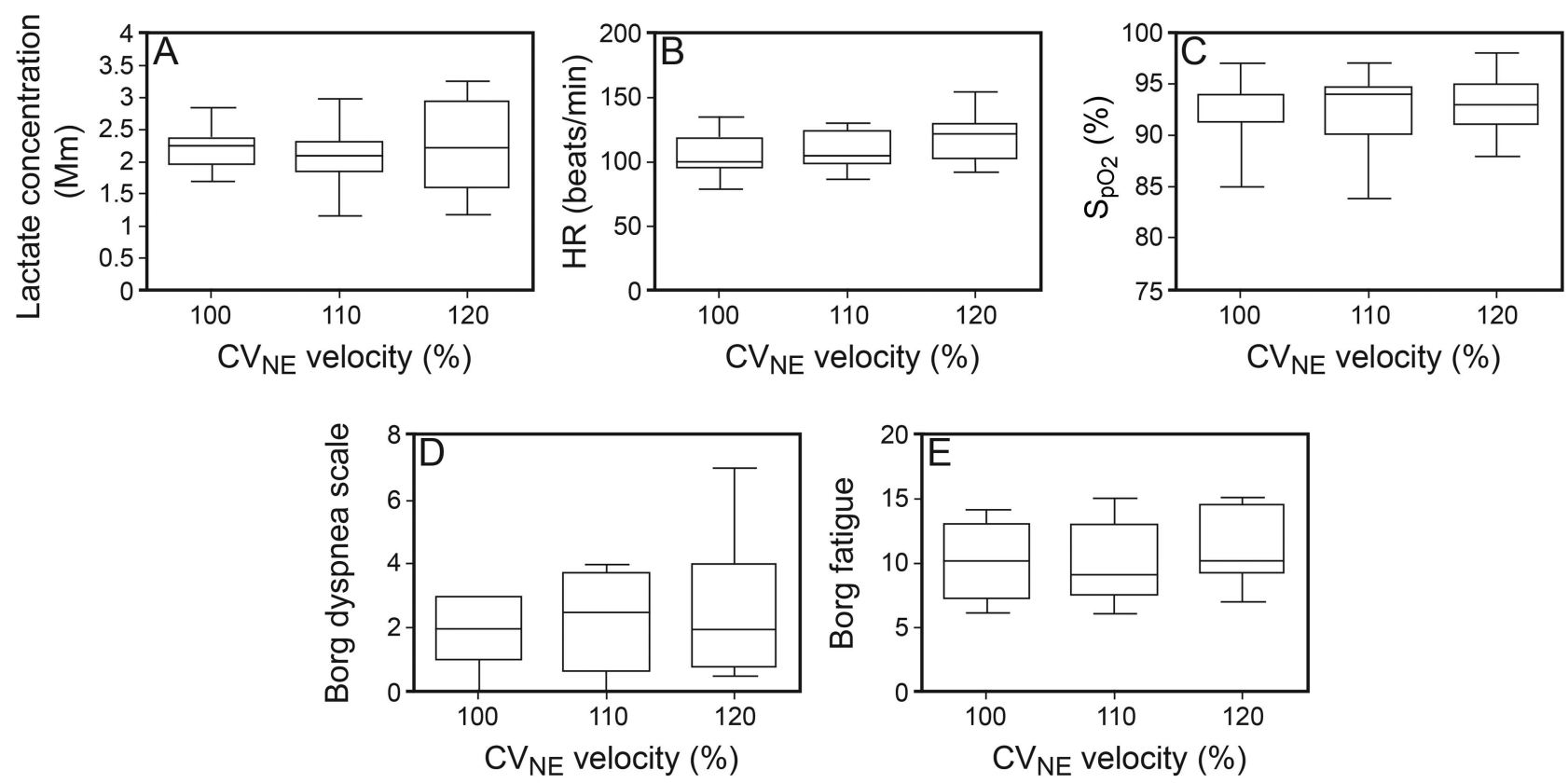

Fig. 3. Box plot of lactate concentration (A), heart rate (B), peripheral oxygen saturation (C), modified Borg scale (D), and Borg fatigue (E) at $100 \%, 110 \%$, and $120 \%$ of critical velocity determined in a non-exhaustive manner for characterization training. Boxes show third and first quartiles, lines represent the median, and whiskers indicate minimum and maximum values.

\section{Phase B: Exercise Tolerance Testing}

We analyzed exercise tolerance testing at $100 \%, 110 \%$, and $120 \%$ of the critical velocity, and we observed that even at intensities of $120 \%$, only 4 subjects $(33 \%)$ had to stop the test due to hemodynamic instability or decline of oxygen saturation, while there was no destabilization in the lactate. Regarding these findings, lactate may not be a good intensity marker for this population because concentrations at rest were very high, which may explain the negative change values. de Souza GF et $\mathrm{al}^{29}$ showed lactate values at rest are $>2 \mathrm{mM}$ in subjects with COPD. The reason for this wide variation in lactate might be due to hypoxemia and low blood flow to the muscles, leading to abnormalities in the oxidative capacity of the skeletal muscles. ${ }^{30,31}$

Another possible explanation for non-destabilization of lactate may be due to the duration of effort during tests. As established by previous studies, ${ }^{12,13}$ we used 2 efforts of $180 \mathrm{~s}$ separated by an interval of $90 \mathrm{~s}$. However, other authors have suggested longer efforts to determine aerobic capacity by lactate (eg, $360 \mathrm{~s}$ ). ${ }^{32}$ Thus, the lower duration of effort used in this study may have underestimated the intensity of the exercise tolerance testing. Furthermore, when the effort is performed in the moderate physiological domain, the lactate reaches a steady state at 120-180s, and at the heavy physiological domain, the lactate is stable after $300-600 \mathrm{~s},{ }^{33}$ and in a severe physiological domain, the lactate increases until the exercise is interrupted without stabilization. ${ }^{33}$
Rossi et al ${ }^{13}$ conducted a study with postmenopausal women in which the participants performed 2 efforts at the same intensity $(4,5$, or $6 \mathrm{~km} / \mathrm{h})$. The authors recommended sessions with individual intensities prescribed according to physical fitness. Thus, the mechanical inefficiency at lower intensities might modify the behavior of lactate and heart rate and compromise $\Delta$ values, consequently compromising $\mathrm{CV}_{\mathrm{NE}}$ determination (eg, negative slope). Concerning this issue, the strength of our study is that the intensities were prescribed individually, aiming to prevent factors that could interfere in the lactate destabilization.

The limitations of this study were the small sample size, which did not allow us to stratify participants by disease severity.

This study has clinical relevance because the proposed method may be a new tool for exercise prescription for patients with COPD. However, we emphasize the need for adaptations concerning the design of the study, especially if the intention is to use it to prescribe aerobic training in this population. This method can be a promising field of study for future research because it prevents the patient from performing at maximum physical exertion.

Suggestions for future studies include longer duration of stages for the determination of aerobic capacity. Furthermore, the validation should be compared with the gold standard method, that is, the cardiopulmonary exercise test.

In conclusion, although most subjects presented good tolerance for exercise at $\mathrm{CV}_{\mathrm{NE}}$, fair levels of reproducibility were observed in subjects with COPD, even using lac- 


\section{Non-Exhaustive Method to Determine Anaerobic Threshold}

tate. Thus, the use of $\mathrm{CV}_{\mathrm{NE}}$ determined through double 3-min efforts should be considered with caution.

\section{REFERENCES}

1. Hill K, Gardiner PA, Cavalheri V, Jenkins SC, Healy GN. Physical activity and sedentary behaviour: applying lessons to chronic obstructive pulmonary disease. Intern Med J 2015;45(5):474-482.

2. Gloeckl R, Marinov B, Pitta F. Practical recommendations for exercise training in patients with COPD. Eur Respir Rev 2013;22(128): 178-186.

3. Hartman JE, Boezen HM, Zuidema MJ, de Greef MH, Ten Hacken $\mathrm{NH}$. Physical activity recommendations in patients with chronic obstructive pulmonary disease. Respiration 2014;88(2):92-100.

4. Wen H, Gao Y, An JY, Chen QL, Zheng JP. [Evaluation of exercise intensity for pulmonary rehabilitation in patients with chronic obstructive pulmonary disease]. Zhonghua Jie $\mathrm{He} \mathrm{He} \mathrm{Hu}$ Xi Za Zhi 2007;30(1):27-30.

5. Reed JL, Pipe AL. Practical approaches to prescribing physical activity and monitoring exercise intensity. Can J Cardiol 2016;32(4): 514-522.

6. Force ERST, Palange P, Ward SA, Carlsen KH, Casaburi R, Gallagher CG, et al. Recommendations on the use of exercise testing in clinical practice. Eur Respir J 2007;29(1):185-209.

7. American Thoracic Society, American College of Chest Physicians. ATS/ACCP Statement on cardiopulmonary exercise testing. Am J Respir Crit Care Med 2003;167(2):211-277.

8. Eaton T, Young P, Nicol K, Kolbe J. The endurance shuttle walking test: a responsive measure in pulmonary rehabilitation for COPD patients. Chron Respir Dis 2006;3(1):3-9.

9. Mador MJ, Modi K. Comparing various exercise tests for assessing the response to pulmonary rehabilitation in patients with COPD. J Cardiopulm Rehabil Prev 2016;36(2):132-139.

10. Weber KT, Janicki JS, McElroy PA, Maskin CS. Cardiopulmonary exercise testing in clinical practice. Cardiology 1987;74(1):62-70.

11. Ferrazza AM, Martolini D, Valli G, Palange P. Cardiopulmonary exercise testing in the functional and prognostic evaluation of patients with pulmonary diseases. Respiration 2009;77(1):3-17.

12. Chassain AP. [Méthode d'appréciation objective de la tolérance de l'organisme à l'effort: application à la mesure des puissances critiques de la fréquence cardiaque et de lalactatémie]. Sci Sports 1986; 1:41-44.

13. Rossi FE, Kalva-Filho CA, de Araújo RG, Gerosa Neto J, Campos EZ, Pastre CM, et al. Critical velocity determined by a non-exhaustive method in menopausal women. Sci Sports 2015;30(1):e17-e22.

14. Papoti M, Zagatto AM, Mendes OC, Gobatto CA. [Utilização de métodos invasivo e não invasivo na predição das performances aeróbia e anaeróbia em nadadores de nível nacional]. Rev Port Cien Desp 2005;5:07-14

15. Zagatto AM, Kalva-Filho CA, Loures JP, Kaminagakura EI, Redkva PE, Papoti M. Anaerobic running capacity determined from the critical velocity model is not significantly associated with maximal ac- cumulated oxygen deficit in army runners. Sci Sports 2013;28(6): e159-e165.

16. Vogelmeier CF, Criner GJ, Martinez FJ, Anzueto A, Barnes PJ, Bourbeau J, et al. Global strategy for the diagnosis, management, and prevention of chronic obstructive lung disease 2017 report: GOLD executive summary. Eur Resp J 2017;49(6):1750214.

17. Lohman TG, Roche AF, Martorell R. Anthropometric standardization reference manual. Champaign: Human Kinetics Books; 1988.

18. Miller MR, Hankinson J, Brusasco V, Burgos F, Casaburi R, Coates A, et al. Standardisation of spirometry. Eur Respir J 2005;26(2):319338.

19. Duarte AAO, Pereira CAC, Rodrigues SCS. [Validação de novos valores previstos brasileiros para a espirometria forçada na raça branca e comparação com os valores previstos obtidos por outras equações de referência]. J Bras Pneumol 2007;33:527-535.

20. ATS statement: guidelines for the six-minute walk test. Am J Respir Crit Care Med 2002;166(1):111-117.

21. Enright PL, Sherrill DL. Reference equations for the six-minute walk in healthy adults. Am J Respir Crit Care Med 1998;158(5):13841387.

22. Borg GAV, Noble BJ. Exercise and sport sciences reviews. New York: Academic Press; 1974:131-57.

23. Hopkins WG. Measures of reliability in sports medicine and science. Sports Med 2000;30(1):1-15.

24. Hazra A, Gogtay N. Biostatistics series module 6: correlation and linear regression. Indian J Dermatol 2016;61(6):593.

25. Cohen J. Statistical power analysis for the behavioral sciences. Hilsdale: Lauwrence Erlbaum Associates Publisher; 1988.

26. Bland JM, Altman DG. Statistical methods for assessing agreement between two methods of clinical measurement. Lancet 1986;1(8476): 307-310.

27. Wasserman K, Whipp BJ, Koyl SN, Beaver WL. Anaerobic threshold and respiratory gas exchange during exercise. J Appl Physiol 1973;35(2):236-243.

28. Caviedes IR, Delgado I, Soto R. Ventilatory inefficiency as a limiting factor for exercise in patients with COPD. Respir Care 2012; 57(4):583-589.

29. de Souza GF, Castro AA, Velloso M, Silva CR, Jardim JR. Lactic acid levels in patients with chronic obstructive pulmonary disease accomplishing unsupported arm exercises. Chron Respir Dis 2010; 7(2):75-82

30. Maltais F, Simard AA, Simard C, Jobin J, Desgagnes P, LeBlanc P. Oxidative capacity of the skeletal muscle and lactic acid kinetics during exercise in normal subjects and in patients with COPD. Am J Respir Crit Care Med 1996;153(1):288-293.

31. Serres I, Hayot M, Prefaut C, Mercier J. Skeletal muscle abnormalities in patients with COPD: contribution to exercise intolerance. Med Sci Sports Exerc 1998;30(7):1019-1027.

32. Kuipers H, Rietjens G, Verstappen F, Schoenmakers H, Hofman G. Effects of stage duration in incremental running tests on physiological variables. Int J Sports Med 2003;24(7):486-491.

33. Burnley M, Doust JH, Vanhatalo A. A 3-min all-out test to determine peak oxygen uptake and the maximal steady state. Med Sci Sports Exerc 2006;38(11):1995-2003 